\title{
Retocolite Ulcerativa (RCU): Perfil Evolutivo Clínico Endoscópico. Estudo Retrospectivo
}

\section{Ulcerative Colitis: Clinical and Endoscopic Profile. A Retrospective Study}

\author{
EDSON JURADO DASILVA ${ }^{1}$; IARAVASCONCELLOS SEIXAS ${ }^{2}$ \\ ${ }^{1}$ Serviço de Coloproctologia do Hospital dos Servidores do Estado-RJ Ministério da Saúde, Serviço de Endoscopia \\ Digestiva da Casa de Portugal RJ e clínica privada; ${ }^{2}$ Serviço de Coloproctologia do Hospital dos Servidores do \\ Estado-RJ Ministério da Saúde.
}

\begin{abstract}
SILVA EJ; SEIXAS IV. Retocolite Ulcerativa (RCU): Perfil Evolutivo Clínico Endoscópico. Estudo Retrospectivo. Rev bras Coloproct, 2008;28(1): 031-035.

RESUMO: Objetivo: Analisar o perfil da RCU. Casuística e Métodos: Análise retrospectiva de pacientes acompanhados pelos autores de 1996 a 2006. Confrontamos topografia das lesões, colonoscopia / histopatologia com ênfase na displasia e CCR. Rastreamos displasia após 7 anos de doença. Teste $t$ de Student foi usado para média e qui-quadrado para números absolutos. $\mathrm{P}<0,05$ significativo. Resultados: 127 pacientes, 40 homens e 87 mulheres. Média de idade $47 \pm 13 \mathrm{~N}=61(48 \%)$ na pancolite e $45 \pm 15$ na colite esquerda $N=66(52 \%)$ p $>0,05$ para idade. Displasia em $9(7 \%), 8(6 \%)$ na pancolite $p<0,01$. Dois $(2 \%)$ em mucosa lisa e $7(12,5 \%)$ em portadores de nodularidade e DALM (dysplasia associated lesion or mass) $\mathbf{p}<0,05$. Pseudopólipos $\mathrm{N}=55(43 \%)$. CCR em 7 (5,5\%). 13 colectomizados $(10 \%), 3$ por intratabilidade, 3 por megacólon tóxico, 1 por displasia com tumor na peça e 6 com CCR. CCR tinham mais de 12 anos de doença. Em $3(2,3 \%)$ com doença ativa à histopatologia não foi detectado sangue macroscópico nas fezes. Conclusões: Reafirmamos a necessidade de vigilância para displasia e CCR. Pancolite aumenta risco de displasia e CCR. A idade não interfere na extensão da doença. Sangue nas fezes é importante no diagnóstico.
\end{abstract}

Descritores: Colite, displasia, câncer, colonoscopia.

\section{INTRODUÇÃO}

A RCU é importante pela sua cronicidade, morbidade e associação com o CCR $\left({ }^{1,2}\right)$. Os fatores de risco para o desenvolvimento do CCR são: tempo e gravidade da doença, história familiar para CCR, associação com colangite esclerosante primária e a presença de displasia $(3,4,5,6,7,8)$.

\section{OBJETIVO}

Analisar o perfil clínico evolutivo da RCU com ênfase no acompanhamento colonoscópico.

\section{PACIENTES E MÉTODOS}

Análise retrospectiva de pacientes acompanhados pelos autores em clínica privada, Serviços de Endoscopia Digestiva da Casa de Portugal e de Coloproctologia do HSE- MS do Rio de Janeiro, no período de 1996 a 2006. Foram incluídas somente as formas típicas da doença. Confrontamos topografia das lesões, colonoscopia/histopatologia com ênfase no diagnóstico de displasia e do CCR. Rastreamos displasia após 7 anos do início ou do diagnóstico da doença. Teste $t$ de Student foi usado para análise da média e desvio padrão, e qui-quadrado para estudo de números absolutos. $\mathrm{P}<$ que 0,05 foi considerado significativo.

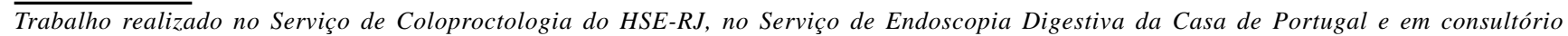
particular - Rio de Janeiro - RJ - Brasil.
} 


\section{RESULTADOS}

Preencheram os parâmetros para este estudo 127 pacientes, sendo 87 do sexo feminino e 40 do sexo masculino. O mais jovem com 18 e o mais velho com 72 anos de idade na data da avaliação.

A Tabela 1 mostra a idade em anos confrontada com a topografia ou estadiamento da doença.

Displasia foi detectada em 9 pacientes, perfazendo um total de $7 \%$ e a Tabela 2 mostra a sua distribuição em função do estadiamento topográfico da doença.

A displasia foi mais freqüente em mucosa não lisa, correspondendo a presença de pseudopólipos ou pólipos inflamatórios, nodularidade e DALM (dysplasia associated lesion or mass) como podemos observar na Tabela 3.

Detectamos a presença de pólipos inflamatórios, pseudopólipos, em 55 (43\%) dos 127 acompanhados.

CCR foi encontrado em 7 pacientes $(5,5 \%)$, todos observados em cólon proximal e em portadores de RCU com mais de 12 anos de evolução. Em um deles, a RCU foi diagnosticada na adolescência e o CCR apareceu após 21 anos de tratamento. na Tabela 4.

A idade nos portadores de CCR pode ser vista

O perfil do CCR na RCU pode ser evidenciado na Tabela 5, que mostra também as indicações de proctocolectomia $\mathrm{N}=13$ (10 \%) na amostra estudada.
Interessante salientar também, que a presença de sangue vivo visível nas fezes correspondeu à doença ativa histopatológica, como podemos observar na Tabela 6.

\section{DISCUSSÃO}

A presença de pancolite é importante na evolução e progressão da RCU e deverá ser topograficamente confirmada por estadiamento histopatológico $\left({ }^{9}\right)$. Neste contexto, a displasia foi mais freqüente em mucosa macroscopicamente doente, isto é, com nodularidade e $D A L M$, como se pode observar nas TABELAS 2 e 3, fato consoante com a literatura especializada $\left({ }^{10}\right)$. Embora menos freqüente, a displasia poderá ser também detectada em mucosa lisa e aparentemente normal. Para aprimorarmos o índice de diagnóstico, devemos aumentar o número de fragmentos obtidos. Costumamos coletar cerca de 30 fragmentos. Eventualmente, usamos cromoscopia com índigo carmin à $0,4 \%$ com aparelho de magnificação, embora na nossa experiência não percebamos vantagem, pois a presença de muco, nem sempre fácil de ser retirado, induz-nos a impressão de se tratar de pitts IV, que significa displasia com CCR invasivo. Parece existir consenso, quando concordamos, que conseguimos melhorar nosso desempenho diagnóstico aumentando o tempo do exame, isto é, trocamos o tempo dispendido no número elevado de biópsias em tempo maior no escrutínio detalhado da mucosa, procurando detalhes em

Tabela 1 - Faixa etária e estadiamento.

\begin{tabular}{lccc}
\hline & Idade & N & \% \\
\hline Pancolite & $47,7 \pm 13$ & 61 & 48 \\
Colite esquerda, inclui proctite ulcerativa & $45,1 \pm 15$ & 66 & 52 \\
P> 0,05 & & 36 & 28,3 \\
Proctite ulcerativa & & 36 \\
\hline
\end{tabular}

Tabela 2 - Displasia e estadiamento topográfico.

\begin{tabular}{lcc}
\hline & $\mathbf{N}$ & $\mathbf{\%}$ \\
\hline Pancolite & 8 & 6,2 \\
Colite esquerda & 1 & 0,8 \\
$\mathrm{P}<0,01$ & & \\
\hline
\end{tabular}

Tabela 3 - Displasia.

\begin{tabular}{lcr}
\hline & N & \% \\
\hline Mucosa lisa & 2 & 2,7 \\
Pseudopólipos, nodularidade e DALM & 7 & 12,5 \\
P $<0,05$ & & \\
\hline
\end{tabular}


Tabela 4 - Faixa etária dos portadores de CCR.

\begin{tabular}{llc}
\hline & $\mathbf{N}$ & \% \\
\hline Idade em anos & $49 \pm 9$ & 9 \\
\hline
\end{tabular}

Tabela 5 - Indicações de proctocolectomia.

\begin{tabular}{lll}
\hline & $\mathbf{N}$ & $\mathbf{\%}$ \\
\hline Intratabilidade & 3 & 2,3 \\
Megacólon tóxico & 3 & 2,3 \\
Displasia multifocal repetida & $1^{*}$ & 0,7 \\
CCR & 6 & 4,7 \\
\hline
\end{tabular}

*No paciente portador de displasia multifocal foi encontrado CCR em área nodular na peça cirúrgica.

anormalidade para com isso dirigir melhor o local das biópsias $\left({ }^{11-12-13}\right)$.

Nem sempre é fácil distinguir pseudopólipo de $D A L M$ e de adenoma. Adenoma costuma se manifestar como pólipo, que geralmente ocorre em mucosa macroscopicamente normal, e deverá ser retirado, sempre que for possível em peça única. O diagnóstico final será histopatológico. Sendo adenoma um epitélio displásico, como tal será rotulado e o tratamento através polipectomia com alça diatérmica está bem definido. DALM, achado característico de doença inflamatória crônica intestinal, sobretudo RCU, se encontra em área doente e às vezes se confunde com pólipo inflamatório ou pseudopólipo, com a diferença de que este não é neoplásico, exibe apenas processo inflamatório característico da RCU, enquanto aquele apresenta-se como lesão displásica elevada, confirmada histopatológicamente quando da sua retirada. DALM, portanto, se encontra em cólon doente, não raro com displasia observada também em outras áreas, multifocal, sendo importante fator na gênese do CCR na RCU e um dos fatores determinantes na indicação de proctocolectomia $\left({ }^{14-15-16}\right)$.
A pesquisa de displasia na RCU é muito importante e deverá ser procurada sempre que possível, em doença inativa, pois o epitélio em reparação pode ser confundido com o displásico. Epitélio displásico em mucosa lisa, menos freqüente, é passível de tratamento endoscópico através a mucosectomia $\left({ }^{17}\right)$. Não temos exemplo de epitélio displásico em mucosa lisa tratado por via endoscópica, até porque nestas circunstâncias só percebemos a presença de displasia quando recebemos o laudo histopatológico. Um de nossos pacientes apresentava displasia multifocal, inclusive em área nodular. Era portador de doença há 21 anos, com controle clínico difícil, e RCU iniciada no começo da adolescência, motivo pelo qual optou-se pela proctocolectomia. Na peça cirúrgica, na área nodular em cólon direito próximo ao ceco, foi diagnosticada a presença também de CCR não identificado macroscopicamente. A indicação cirúrgica neste cenário de displasia não é, no entanto, consenso $\left({ }^{15-18-19}\right)$. As nossas indicações cirúrgicas estão contempladas na TABELA 5.

A incidência de CCR na RCU vem diminuindo ${ }^{(20)}$, provavelmente devido ao melhor aproveitamento do arsenal terapêutico. Tivemos $7(5,5 \%)$ pacientes portadores de CCR, (TABELA 5) com média de idade 49 \pm 9 , (TABELA 4), que está abaixo do esperado quando se trata do CCR esporádico. Embora faça parte da nossa rotina atual, não tivemos computado o histórico familiar com relação ao CCR na amostragem estudada.

A presença de sangue vivo nas fezes em portadores de RCU foi parâmetro estudado e é fundamental para seu diagnóstico, e a TABELA 6 confirma esta assertiva, quando mostra, que doença ativa confirmada por exame histopatológico, está associada a este achado em 97,6\% dos pacientes.

Temos por rotina avaliar nossos pacientes com o toque retal, pesquisando também o aspecto macroscópico das dejeções, pois como já foi descrito $\left({ }^{21}\right)$, o doente portador de RCU, seria um fronteiriço entre a normalidade psicológica e a esquizofrenia, e,

Tabela 6 - Histopatologia e presença de sangue vivo nas fezes.

\begin{tabular}{lrr}
\hline & $\mathbf{N}$ & $\mathbf{\%}$ \\
\hline RCU ativa à histologia e presença de sangue macroscópico nas fezes & 124 & 97,6 \\
RCU ativa à histologia sem a presença de sangue macroscópico nas fezes & 3 & 2,3 \\
$\mathrm{P}<0,01$ & & \\
\hline
\end{tabular}


Rev bras Coloproct Janeiro/Março, 2008
Retocolite Ulcerativa (RCU): Perfil Evolutivo Clínico Endoscópico. Estudo Retrospectivo

Edson Jurado da Silva e Cols.
Vol. 28 $\mathbf{N}^{\mathbf{0}} 1$ portanto, esconderia ou negaria a doença, dificultando assim o diagnóstico. Tivemos oportunidade de confirmar esta afirmativa por várias vezes ao longo dos anos durante exame proctológico sem preparo do cólon, onde além do processo inflamatório difuso próprio da RCU encontramos apenas sangue em meio a poucas fezes líquidas, em alguém que refere somente apresentar diarréia sem sangue. Tenho há muitos anos por hábito, quando examino paciente do sexo masculino, com hematócrito abaixo de 30 e história de diarréia crônica, sem sangue, pensar como primeira hipótese o diagnóstico de RCU, que começa a se delinear após o toque, estando o dedo enluvado sujo de sangue.

\section{CONCLUSÕES}

1. Pancolite com a presença de mucosa não lisa, pseudopólipos, nodularidade e DALM, aumentam significativamente o risco de displasia e de CCR em portador de RCU.

2. Displasia, sobretudo multifocal, é fator determinante para a presença de CCR.

3. A idade não interfere na extensão ou estadiamento topográfico da doença.

4. Sangue vivo nas fezes é determinante no diagnóstico de RCU ativa e deverá ser procurado independente do relato histórico do paciente.

ABSTRACT: Objectives: Ulcerative colitis is a risk factor for colorectal cancer CRC. The aim of this study was to evaluate the profile of this disease. Methods: Retrospective data of patients with ulcerative colitis observed between 1996 and 2006 were analyzed for colonoscopic/histological findings. Colonoscopy surveillance started 7 years after disease onset. Student $t$ test was used for means and chi-square to compare frequency. A p $<0.05$ was considered significant. Results: 127 patients were enrolled in this study, being 40 male and 87 female. Mean age was $47 \pm 13$ years $N=61(48 \%)$ for pancolitis, $45 \pm 15$ years $N=66(52 \%)$ for left side colitis p>0,05 for age. Dysplasia was present in $9(7 \%)$ being $8(6 \%)$ in pancolitis $p<0,01$. Among displastic patients $2(2 \%)$ had it in flat mucosa and $7(\mathbf{1 2 , 5 \%})$ in nodularity and DALM (dysplasia associated lesion or mass) $\mathbf{p}<0,05$. Post-inflammatory polyps were seen in $55(43 \%)$. CRC was diagnosed in $7(5,5 \%) .13(10 \%)$ underwent proctocolectomy, 3 for refractory disease, 3 for toxic megacolon, 1 for dysplasia with tumor diagnosed only at the surgical specimen and 6 for CRC. All of the CRC patients had disease for more than 12 years. Macroscopic blood on stools was seen in all but $3(2 \%)$ with active disease by histology $p<0,01$. Conclusions: Ulcerative colitis is a risk factor for dysplasia and CRC in longstanding extensive disease with abnormal looking mucosa at colonoscopic surveillance. Age does not interfere with extension of disease. Blood means active disease.

Key words: Colitis, dysplasia, cancer, colonoscopy.

\section{REFERÊNCIAS}

1. Lakatos L, Mester G, Erdelyi Z, David G, Pandur T, Balogh $\mathrm{M}$ et al. Risk factors for ulcerative colitis-associated colorectal cancer in a Hungarian cohort of patients with ulcerative colitis: results of a population-based study. Inflamm bowel Dis 2006;12:205-11.

2. van Hogezand RA, Eichhorn RF, Choudry A, Veenendaal RA, Lamers CB.Malignancies in inflammatory bowell disease: fact or fiction? Scand J Gastroenterol Suppl 2002;236:48-2.

3. Goldblum JR. The histologic diagnosis of dysplasia, dysplasiaassociated lesion or mass, and adenoma: a pathologist' $\mathrm{s}$ perspective. J Clin Gastroenterol 2003;36 (5 Suppl):S 63-9.

4. Itzkowitz SH, Harpaz N. Diagnosis and management of dysplasia in patients with inflammatory bowel diseases. Gastroenterology 2004;126:1634-48.

5. Loftus EV. Epidemiology and risk factors for colorectal dysoplasia and cancer in ulcerative colitis. Gastroenterol Clin North Am 2006;35:517-31.
6. McKenna BJ, Appelman HD. Dysplasia can be a pain in the gut. Pathology 2002;34:518-28.

7. Riegler G, Bossa F, Caserta L, Pera A, Tonelli F, Stumiolo $\mathrm{GC}$ et al. Colorectal cancer and high grade dysplasia complicating ulcerative colitis in Italy. A retrospective co-operative IG-IBD study. Dig Liver Dis 2003;35:62834.

8. Ullman T, Croog V, Harpaz N, Sachar D, Itzkowitz S. Progression of flat low-grade dysplasia to advanced neoplasia in patients with ulcerative colitis. Gastroenterology 2003;125:1311-9.

9. Mathy C, Schneider K, Chen YY, Varma M, Terdiman JP, Mahadevan U. Gross versus microscopic pacolitis and the occurrence of neoplasia in ulcerative colitis. Inflamm Bowell Dis 2003;9:351-5.

10. Rutter MD, Saunders BP, Wilkinson KH, Rumbles S, Schofield G, Kamm MA et al. Cancer surveillence in longstanding ulcerative colitis: endoscopic appearances help predict cancer risk. Gut 2004;53:1724-5. 
11. Rutter MD, Saunders BP, Schofield G, Forbes A, Price AB, Talbot IC. Pancolonic indigo carmine dye spraying for the detection of dysplasia in ulcerative colitis. Gut 2004;53:25660

12. Rutter MD, Saunders BP, Wilkinson KH, Kamm MA, Williams CB, Forbes A. Most dysplasia in ulcerative colitis is visible at colonoscopy. Gastrointest Endosc 2004;60:334-9.

13. Toruner M, Harewwood GC, Loftus EV, Sandborn WJ, Tremaine WJ, Faubion WA et al Endoscopic factors in the diagnosis of colorectal dysplasia in chronic inflammatory bowel disease. Inflamm Bowel Dis 2005;11:428-34.

14. Friedman S, Odze RD, Farraye FA. Management of neoplastic polyps in inflammatory bowel disease. Inflamm Bowell Dis 2003;9:260-6.

15. Odze R. Diagnostic problems and advances in inflammatory bowel disease. Mod Pathol 2003;16:347-58.

16. Torres C, Antonioli D, Odze RD. Polypoid dysplasia and adenomas in inflammatory bowel disease: a clinical, pathologic, and follow-up study of 89 polyps from 59 patients. Am J Surg Pathol 1998;22:275-84.

17. Hursltone DP, Sanders DS, Atkinson R, Hunter MD, McAlindon ME, Lobo AL et al. Endoscopic mucosal resection for flat neoplasia in chronic ulcerative colitis: can we change the endoscopic management paradigm? Gut 2007;56:838-46.

18. Befrits R, Ljung T, Jaramillo E, Rubio C. Low-grade dysplasia in extensive, long-standing inflammatory bowel disease: a follow-up study. Dis Colon Rectum 2002;45:615-20.

19. Ekbom A Motion- colonoscopic surveillence is more cost effective than colectomy in patients with ulcerative colitis: argument against the motion. Can J Gastroenterol 2003;17:1224.

20. Rubio CA, Befrits R, Ljung T, Jaramillo E, Slezak P. Colorectal carcinoma in ulcerative colitis is decreasing in Scandinavian countries. Anticancer Res 2001;21:2921-4.

21. Karush. Daniels. Flood. O'Connor. Psychoterapy in chronic ulcerative colitis.Philadelphia ( PA ); W B Saunders Company; 1977.

\section{Endereço para correspondência:}

Rua Rodolfo de Souza 105 - Vila Isabel

Rio de Janeiro, RJ, Brasil

CEP: 20551-270

Tel/Fax: 21 2520-5115

E-mail: edsonjurado@alternex.com.br 